\title{
Temporal signatures of auditory verbal hallucinations : An app-based experience sampling study
}

\section{Bless, Josef J.}

2020-01

Bless , J J , Hjelmervik , H, Torsheim , T , Gudmundsen, M , Laroi , F , Holma , I , Arola , A , Korkeila , J , Hirnstein , M , Marquardt , L, Kusztrits , I, Smelror , R E , Agartz , I \& Hugdahl , K 2020 , ' Temporal signatures of auditory verbal hallucinations : An app-based experience sampling study ' , Schizophrenia Research , vol. 215 , pp. 442-444 . https://doi.org/10.1016/j.schres.2019

http://hdl.handle.net/10138/325517

https://doi.org/10.1016/j.schres.2019.11.020

publishedVersion

Downloaded from Helda, University of Helsinki institutional repository.

This is an electronic reprint of the original article.

This reprint may differ from the original in pagination and typographic detail.

Please cite the original version. 
Letter to the Editor

Temporal signatures of auditory verbal hallucinations: An app-based experience sampling study

\section{Introduction}

The assessment of auditory verbal hallucinations (AVHs) has traditionally been conducted through (semi-)structured interviews, such as the Positive and Negative Syndrome Scales (PANSS; Kay et al., 1987) and the Psychotic Symptom Rating Scales (Haddock et al., 1999) in patients and the Launay Slade Hallucination Scale (Launay and Slade, 1981; Larøi and Van der Linden, 2005) in the general population. However, since standard assessments rely on retrospective and summative reports and thus on the person's accurate memory and capability to estimate the average experience of past hallucination episodes (usually a summary of the last week), the extent to which AVHs are stable or vary across the course of hours or days is currently unknown. To fill this knowledge gap, the current study used an in-house developed mobile application (app) to capture AVHs as experienced in the moment in which they occur. This approach has recently been termed "digital phenotyping" (Insel, 2017; Torous and Keshavan, 2018).

The aim of the present study was to explore the relationships between five key AVH-dimensions (cognitive control, emotional content, spatial localization, intensity, and severity) and for the first time, the temporal patterns of these dimensions.

\section{Method}

Twenty patients ( 12 males, 8 females, mean age: 35.5 years; 10 Norwegian patients; 10 Finnish patients) with a diagnosis of schizophrenia (ICD-10; F20) and who reported experiencing hallucinations (according to PANSS item P3 Hallucinations) were recruited for the study. The data collection was set to a maximum of four weeks. However, in many cases it was shorter (see Fig. S1). The study was approved by the regional ethical committees responsible for the respective location of the data collection. Participants were instructed to report about their AVHs in the moment in which they occur (event-related design). To do this, participants used the in-house developed app which consisted of a series of five questions, each representing a separate AVH-dimension (see Fig. 1). Participants responded on a visual analogue scale (VAS) by moving their finger on a horizontal slider. The score for each answer ranged from zero at the far left of the VAS and continuously increasing to one at the far right of the VAS.

\section{Results}

The average number of days that participants reported on their hallucinations was 19.5 days ( $\max 30$; $\min 6$ ). The average number of sessions per participant was 74.8 sessions ( $\min 24$, max 162 ), of which
$64.2 \%$ were completed. $69.4 \%$ of the completed sessions were initiated by the patient.

The results from a random intercept general linear mixed model analysis showed that all AVH-dimensions were significantly related to each other except for the localization dimension, which was only related to the intensity dimension (see Table S1). The direction of the relationships revealed that, when AVHs had more positive content, they were also experienced as less disturbing, more controlled, and quieter. Moreover, more cognitive control of the AVHs was related to less disturbing and quieter AVHs. Finally, more internal AVHs were associated with quieter AVHs (see Fig. S2).

Also, with regard to temporal effects, a significant interaction between the VAS index-score and day-of-week $[\mathrm{F}(24,1808)=1.72, p=$ .017] was found. Post-hoc tests (Fisher's LSD, $\mathrm{p}<.05$ ) revealed that the interaction was driven by (a) the emotional content dimension, that is, significantly more positive AVHs on Fridays compared to Mondays ( $p=.006)$, Tuesdays ( $p=.041)$, and Thursdays $(p=.026)$, and (b) the severity dimension, that is, significantly more disturbing AVHs on Saturdays compared to Tuesdays $(p=.008)$, Thursdays $(p=.024)$, and Fridays $(p=.001)$. In addition, a significant interaction was observed between the VAS index-score and time-of-day $[\mathrm{F}(12,1762)=$ $1.79, p=.045]$. This interaction was driven by AVHs being rated as louder in the morning ( $6 \mathrm{am}-12 \mathrm{am})$ compared to the afternoon (12 am-6 pm; $p=.005)$ as well as compared to the evening ( $6 \mathrm{pm}-$ $12 \mathrm{pm} ; p=.009)$.

\section{Discussion}

The present study showed for the first time that AVH-dimensions fluctuate between short time-intervals (within hours) and in doing so revealing effects of day-of-the-week and time-of-day.

Our finding that more positive AVHs are associated with more control over AVHs and less distress is in line with previous research which showed that content and control are important factors in differentiating between individuals experiencing AVHs in the general population (without a psychiatric diagnosis) and patients experiencing AVHs (with a psychiatric diagnosis) (Daalman et al., 2011; Johns et al., 2014). It appears that when AVHs are more controllable, they are more likely to have positive content, and this may be a marker of nonclinical AVHs. Conversely, the negative emotional valence of experienced AVHs in clinical populations may have an origin in lack of cognitive control, perhaps due to weak prefrontal inhibition (for a review see Larøi et al., 2019).

The day-of-the-week effect is in line with research on mood in healthy individuals. In a large scale-survey study $(N=340,000)$, Stone et al. (2012) reported strong support for what is called the "Thank God it's Friday" effect, that is, mood tends to be better on Fridays and Saturdays than during the rest of the week. Similar to the findings in the present study, albeit for symptoms of paranoia and negative/positive affect, Oorschot et al. (2012) showed that day-to-day fluctuations were related to contextual factors such as being alone vs. being with 

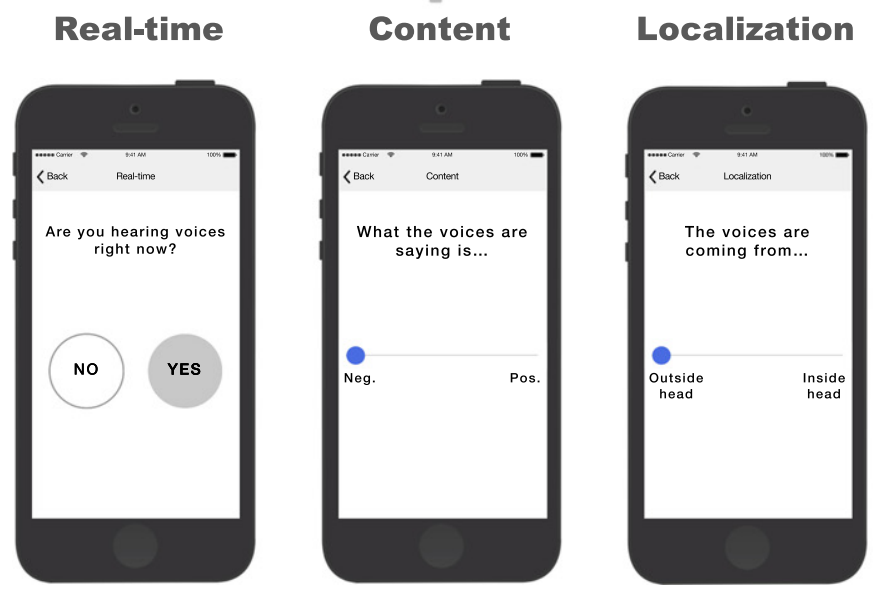

Control

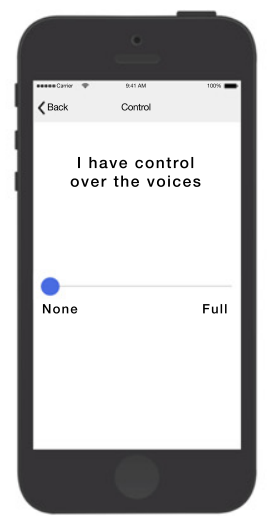

Intensity

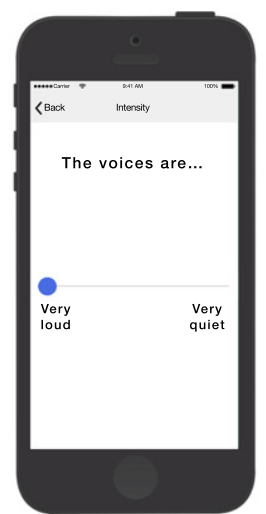

Severity

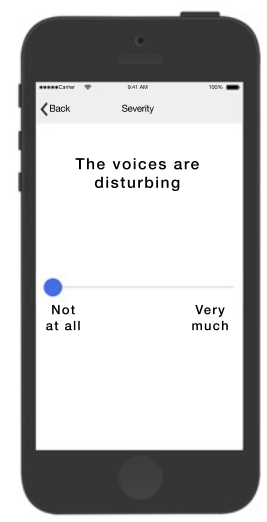

Fig. 1. Illustration of the app containing the initial question and the five questions about AVH-dimensions. (iPhone frames adopted from Todd Zaki Warfel)

family. Together these findings suggest that the current mood state is manifested in the content of the AVH.

With regard to the time-of-day effect, it could be speculated that louder AVHs occur in the morning because this is usually before medication is taken. We also found that AVHs are perceived to come from an external source with increasing intensity (loudness). This suggests that perceived loudness of an AVH may play a crucial role in the misattribution error, that is, internally generated cognitive events are falsely attributed to an external source (see Woodward and Menon, 2013). Along this line, a study by CuevasYust (2014) found that schizophrenia patients experiencing AVHs also reported hearing ordinary thoughts at the same volume as their AVHs and spoken words. In contrast, healthy participants and schizophrenia patients without AVHs reported spoken words as louder than their own thoughts. In this respect, perceived loudness has been linked to reduced activation in distributed brain networks involved in inner speech processes (Vercammen et al., 2011).

Supplementary data to this article can be found online at https://doi. org/10.1016/j.schres.2019.11.020.

\section{Role of the funding source}

This work was supported by research grants awarded to Kenneth Hugdahl from the European Research Council (Advanced Grant, \#693124), the Western Norway Regional Health Authority (\#912045), and the Research Council of Norway (NORMENT \#223273; FRIMEDBIO \#221550), as well as by the Bergen Research Foundation to Marco Hirnstein (BFS2016REK03).

\section{Contributors}

J.J.B. and K.H. designed the study. J.J.B. conducted the literature searches, conducted the statistical analysis, wrote and edited the manuscript. K.H., F.L., and M.H. edited the manuscript. J.J.B. and M.G. developed the mobile application. M.G. programmed the mobile application. H.H. and T.T. assisted with statistical analysis and data interpretation. A.A., L.M., I.K, and R.E.S. conducted the recruitment and data collection. I.H., J.K., and I.A. established contacts necessary for recruitment. All authors contributed to and have approved the final manuscript.

Declaration of competing interest

The authors report no conflicts of interest.

\section{Acknowledgement}

The authors would like to thank all of the participants in this study.

\section{References}

Cuevas-Yust, C., 2014. Do thoughts have sound? Differences between thoughts and auditory hallucinations in schizophrenia. Span. J. Psychol. 17, E27.

Daalman, K., Boks, M.P., Diederen, K.M., de Weijer, A.D., Blom, J.D., Kahn, R.S., Sommer, I.E., 2011. The same or different? A phenomenological comparison of auditory verbal hallucinations in healthy and psychotic individuals. J. Clin. Psychiat. 72, 320-325.

Haddock, G., McCarron, J., Tarrier, N., Faragher, E.B., 1999. Scales to measure dimensions of hallucinations and delusions: the psychotic symptom rating scales (PSYRATS). Psychol. Med. 29, 879-889.

Insel, T.R., 2017. Digital phenotyping: technology for a new science of behavior. JAMA $318,1215-1216$.

Johns, L.C., Kompus, K., Connell, M., Humpston, C., Lincoln, T.M., Longden, E., Preti, A., Alderson-Day, B., Badcock, J.C., Cella, M., Fernyhough, C., McCarthy-Jones, S., Peters, E., Raballo, A., Scott, J., Siddi, S., Sommer, I.E., Laroi, F., 2014. Auditory verbal hallucinations in persons with and without a need for care. Schizophr. Bull. 40 (Suppl. 4), S255-S264.

Kay, S.R., Fiszbein, A., Opler, L.A., 1987. The positive and negative syndrome scale (PANSS) for schizophrenia. Schizophr. Bull. 13, 261-276.

Larøi, F., Van der Linden, M., 2005. Normal subjects' reports of hallucinatory experiences. Can. J. Behav. Sci. 37, 33-43.

Larøi, F., Thomas, N., Aleman, A., Fernyhough, C., Wilkinson, S., Deamer, F., McCarthyJones, S., 2019. The ice in voices. Understanding negative content in auditory-verbal hallucinations. Clin. Psychol. Rev. 67, 1-10.

Launay, G., Slade, P., 1981. The measurement of hallucinatory predisposition in male and female prisoners. Personal. Individ. Differ. 2, 221-234.

Oorschot, M., Lataster, T., Thewissen, V., Bentall, R., Delespaul, P., Myin-Germeys, I., 2012. Temporal dynamics of visual and auditory hallucinations in psychosis. Schizophr. Res. $140,77-82$.

Stone, A.A., Schneider, S., Harter, J.K., 2012. Day-of-week mood patterns in the United States: on the existence of 'Blue Monday', 'Thank God it's Friday' and weekend effects. J. Posit. Psychol. 7, 306-314.

Torous, J., Keshavan, M., 2018. A new window into psychosis: the rise digital phenotyping, smartphone assessment, and mobile monitoring. Schizophr. Res. 197, 67-68.

Vercammen, A., Knegtering, H., Bruggeman, R., Aleman, A., 2011. Subjective loudness and reality of auditory verbal hallucinations and activation of the inner speech processing network. Schizophr. Bull. 37 (5), 1009-1016.

Woodward, T.S., Menon, M., 2013. Misattribution models (II): source monitoring in hallucinating schizophrenia subjects. In: Jardri, R., Cachia, A., Thomas, P., Pins, D. (Eds.), The Neuroscience of Hallucinations. Springer, New York, pp. 169-184.

Josef J. Bless Department of Biological and Medical Psychology, University of Bergen, Bergen, Norway NORMENT Center of Excellence, Haukeland University Hospital, Bergen, Norway

Corresponding author at: Department of Biological and Medical Psychology, University of Bergen, Jonas Lies vei 91, 5009 Bergen, Norway.

E-mail address: josef.bless@uib.no

Helene Hjelmervik Department of Biological and Medical Psychology, University of Bergen, Bergen, Norway 
NORMENT Center of Excellence, Haukeland University Hospital, Bergen, Norway

Torbjørn Torsheim Department of Psychosocial Science, University of Bergen, Bergen, Norway

Magne Gudmundsen Department of Biological and Medical Psychology, University of Bergen, Bergen, Norway

Frank Larøi Department of Biological and Medical Psychology, University of Bergen, Bergen, Norway NORMENT Center of Excellence, Haukeland University Hospital, Bergen, Norway

Psychology and Neuroscience of Cognition Research Unit, University of Liège, Liège, Belgium

Irina Holma Mental Health Unit, National Institute for Health and Welfare, Helsinki, Finland Department of Psychiatry, University of Helsinki and Helsinki University Hospital, Helsinki, Finland

Anne Arola

Department of Psychology and Logopedics, Faculty of Medicine, University of Helsinki, Helsinki, Finland

Jyrki Korkeila

Department of Psychiatry, University of Turku, Turku, Finland Satakunta Hospital District, Pori, Finland

Marco Hirnstein Department of Biological and Medical Psychology, University of Bergen, Bergen, Norway NORMENT Center of Excellence, Haukeland University Hospital, Bergen, Norway
Lynn Marquardt Department of Biological and Medical Psychology, University of Bergen, Bergen, Norway NORMENT Center of Excellence, Haukeland University Hospital, Bergen, Norway

Isabella Kusztrits Department of Biological and Medical Psychology, University of Bergen, Bergen, Norway NORMENT Center of Excellence, Haukeland University Hospital, Bergen, Norway

Runar E. Smelror NORMENT, KG Jebsen Center for Psychosis Research, Institute of Clinical Medicine, University of Oslo, Oslo, Norway

Department of Psychiatric Research, Diakonhjemmet Hospital, Oslo, Norway

Ingrid Agartz

NORMENT, KG Jebsen Center for Psychosis Research, Institute of Clinical Medicine, University of Oslo, Oslo, Norway Department of Psychiatric Research, Diakonhjemmet Hospital, Oslo, Norway

Centre for Psychiatric Research, Department of Clinical Neuroscience, Karolinska Institutet, Stockholm, Sweden

Kenneth Hugdahl Department of Biological and Medical Psychology, University of Bergen,

Bergen, Norway NORMENT Center of Excellence, Haukeland University Hospital, Bergen, Norway Division of Psychiatry, Haukeland University Hospital, Bergen, Norway

7 February 2019 